\title{
EINE NEUE KARTE DER ALPENLÄNDER
}

\author{
EDUARD IMHOF
}

"Warum - so wurde ich oft gefragt - verwenden Sie in den Länderkarten kleiner Maßstäbe Ihrer Schulatlanten immer noch die veraltete Schraffenmethode und die so unnatürlichen braunen Höhenfarbtöne? Was hindert Sie daran, auch in diesen Karten die naturähnlichere, anschaulichere farben- und schattenplastische Darstellung Ihrer Karten großer Maßstäbe zur Anwendung zu bringen?»

Solche Fragen überraschten mich keineswegs. Eine Modernisierung der Geländedarstellung in Karten kleiner Maßstäbe war längst fällig. Das Problem beschäftigte seit Jahren nicht nur mich, sondern manchen Kartographen manchen Landes. Der Weg zur Lösung aber war dornenvoll. Er führte durch die Wirrnisse grundsätzlicher Auseinandersetzungen über konkrete und abstrakte Darstellung, er führte durch das Gestrüpp alter, festeingewurzelter Lehrmeinungen und Gewohnheiten und er mußte geebnet werden durch einige wesentliche Verbesserungen und Verfeinerungen reproduktionstechnischer Dinge und Vorgänge. -

Heute endlich sind wir soweit, daß in unseren Schulatlanten der Übergang von der alten zur neuen Lösung verantwortet und empfohlen werden kann. -

Vor einigen Jahren beauftragte mich die Konferenz der Kantonalen Erziehungsdirektoren, den Schweizerischen Mittelschulatlas einer Erneuerung zu unterziehen. (Siehe darüber Lit. Nr. 5.) Im Rahmen dieser Umformung drängte sich auch die oben angedeutete Neugestaltung der Geländezeichnung auf.

Der Schweizerische Mittelschulatlas, wie auch sein kleinerer Bruder, der Schweizerische Sekundarschulatlas, sind in technischer Hinsicht Gemeinschaftswerke des Verfassers dieses Aufsatzes und der Art. Institut Orell Füßli AG. in Zürich. Seit manchem Jahr bemühen wir uns gemeinsam um die neue Kartenform. Nach anfänglichen Schwierigkeiten und Enttäuschungen führten diese Bemühungen endlich zu Ergebnissen, die unseren Wünschen weitgehend entsprechen. Ein Beispiel der neuen Lösung zeigt die Karte der Alpenländer 1:2500 ooo der im Frühjahr 1959 erscheinenden Ausgabe des Schweizerischen Sekundarschulatlas (Lit. Nr. 3). Ein nur das Gewässernetz und das Gelände enthaltender Sonderdruck dieser Karte ist dem vorliegenden Heft der «Geographica Helvetica» beigegeben.

Im Folgenden seien die verschiedenen Aspekte der neuen Lösung betrachtet, die frühere und die neue Lösung seien einander gegenübergestellt. Die bisherige Karte dürfte den meisten Lesern leicht zugänglich sein, sie findet sich in den früheren Ausgaben sowohl des Schweiz. Mittelschulatlas (Lit. Nr. 2), wie des Schweiz. Sekundarschulatlas.

\section{1) Die bisherige Darstellung}

Die bisherige Karte gibt die Geländeformen in traditioneller Weise durch braune oder graubraune Schraffen. Entsprechend einer angenommenen Modell-Schrägbeleuchtung aus Nordwesten sind in höheren, stark durchtalten Gebirgen die Lichthänge leichter, die Schattenhänge kräftiger schraffiert. Damit entsteht ein gewisser Reliefeindruck. Die Höhenlagen sind durch die sieben folgenden Flächenfarbtöne zum Ausdruck gebracht:

$\begin{aligned} 0-100 \mathrm{~m} & \text { Blaugrün } \\ 100-200 \mathrm{~m} & \text { leichtes, gelbliches Grün } \\ 200-500 \mathrm{~m} & \text { Gelb } \\ 500-1000 \mathrm{~m} & \text { leichtes, gelbliches Braun } \\ 1000-2000 \mathrm{~m} & \text { Braun } \\ 2000-4000 \mathrm{~m} & \text { Braunrot } \\ \text { über } 4000 \mathrm{~m} & \text { kräftiges Braunrot }\end{aligned}$


Diese Abstufung ist geometrisch, wie topographisch oder geomorphologisch durchaus zweckmäßig. Die Stufengrenzen entsprechen sehr einfachen, einprägsamen Zahlen. Die Stufen wachsen stetig, und zwar angenähert im Sinne einer geometrischen Progression. Die Progression entspricht in großen Zügen der hypsometrischen Kurve der festen Erdoberfläche. Danach erweisen sich als zweckmäßig kleine Stufen für die ausgedehnten, klimatisch und kulturgeographisch reich differenzierten Tiefländer, große Stufen aber für die regional eng begrenzten und vorwiegend durch Schraffen zum Ausdruck gebrachten Hochgebirge.

In der Wahl der Farben entspricht die Skala gleichsam einer Zusammensetzung von zwei Hell-Dunkel-Reihen oder Intensitätsreihen:

Von einem hellen, gelben Mittelton aus (in der Region 200 bis $500 \mathrm{~m}$ ) verdunkeln sich die blaugrünen Töne nach unten, die braunen nach oben. Eine solche zweifache Hell-Dunkelabstufung ergibt deutlich unterscheidbare Farben; das Tieflandgrün symbolisiert recht gut die fruchtbaren Ebenen, das dazu gegensätzliche Höhenbraun aber das vegetationsärmere Gebirge. Anklänge an gewisse Natureffekte, wie z. B. an luftperspektivische Landschaftseffekte, sind solchen Farbfolgen nicht ganz abzusprechen. Trotzdem handelt es sich im Wesentlichen um symbolische, abstrakte Farbtöne, um eine gut differenzierte Zweckmäßigkeitsskala. Solche oder ähnliche Höhenreihen haben sich seit Jahrzehnten in den Länderkarten kleiner Maßstäbe eingelebt, sie sind heute weit verbreitet. (In jüngster Zeit freilich ersetzte man das Höhenbraun da und dort durch graue oder violette Töne, was aber kaum zur Kartenverschönerung beigetragen hat.)

Diese traditionelle Farbskala leidet an zwei schwerwiegenden Mängeln:

Erstens: Eine mittlere Stufe, diejenige von 200-500 m, hebt sich als hellste Zone aus dem Kartenbild heraus. Ausgehend von dieser Mittellage stufen sich die Farbtöne ab im Sinne der beiden, sich widersprechenden Relationen «je dunkler, desto höher» und «je dunkler, desto niedriger». Dies entbehrt einer gewissen, auch beim graphischen Gestalten nicht ausseracht zu lassenden Logik. -

Ein zweiter Einwand ist schwerwiegender:

Die kräftigen braunen und rotbraunen Höhenfarben schwächen den Reliefeindruck der Schraffen, ja sie verwischen das Geländebild fast völlig.

Die Alpen, in der Natur ein großartiges, reich gegliedertes, tief durchfurchtes und scharfkantiges Riesenrelief, erscheinen in der Karte infolge der gegenseitigen Überlagerung brauner Schraffen und ebenfalls brauner, z. T. gerasterter Höhen-Farbflächen als eine wüste, ungegliederte Masse, so wie am ersten Schöpfungstage, als sie noch nicht durch das Licht erhellt waren. Man kann solche abstrakten Erdoberflächenbilder wohl kartenleserisch entziffern oder ausdeuten, doch kann man die Reliefformen nicht unmittelbar sehen. Für solche Karten gilt heute noch die Auffassung des bekannten Geographen OsKar Peschel, der einst schrieb: «Landkarten sind Steine der Weisen, sie sind aber auch nichts weiter als Steine, wenn ihnen der Weise mangelt. Landkarten sind Sinnbilder, die in einer Geheimsprache zu uns reden.» (Lit. Nr.6 und 7.)

Schlimm ist, daß das Schraffenrelief gerade in den höheren Zonen, in den braunen Höhenstufen, am meisten verschleiert wird, während es in der tiefern, weniger bewegten Gelbstufe zwischen 200 und 500 m ungestört zur Geltung kommt. -

\section{2) Die neue Darstellung}

In der neuen Karte ist das Schraffenbild ersetzt durch Schattentöne. Man denke sich ein formähnliches Modell des Gebirges und schräg aus Nordwesten auf dessen Oberfläche fallendes Licht. Die so entstehende Hell-Dunkel-Modulation von oben betrachtet oder abgebildet, ergäbe ein unmittelbares Bild der Reliefoberflächenform. Die vorliegende Karte der Alpen, obgleich zeichnerisch entstanden, entspricht weitgehend einem solchen Reliefschattenbild. 
Diese sogenannte "Schattenplastik» allein aber genügt im vorliegenden Falle nicht. Sie würde wohl die Zerknitterungen der Oberfläche, die Taleinschnitte und Bergkämme zeigen, nicht aber die verschiedenen Höhenlagen weit auseinander gelegener Geländeteile. Die Oberrheinische Tiefebene in der Gegend von Mannheim erschiene gleich hoch, wie deren oberes Ende bei Mühlhausen und Basel. Daher kann auch die neue Karte nicht auf Farbtöne für die verschiedenen Höhenstufen verzichten. Die Stufenhöhen oder Stufenabgrenzungen sind dieselben, wie in der alten Karte, die Stufenfarben aber völlig verschieden. Sie sind dem farbigen Aspekt der Natur, der von fern betrachteten Landschaft angenähert. Vor allem aber sind sie, um die Reliefmodulation zu schonen, möglichst hell gehalten. Ihre Abstufung entspricht dem Grundsatz «je höher, desto heller». Das kräftige Braun und Braunrot der Höhen ist durch Gelb und Weiß ersetzt. Damit wurde eine Schwächung der schattenplastischen Effekte nicht nur vermieden, sondern es wurden im Gegenteil diese Effekte unterstützt durch eine gewisse Farben- oder Höhenplastik.

- In den Jahren 1898-1910 entwickelte KarL Peucker (Lit. Nr. 8 und 9) seine, in der Kartographie langezeit als revolutionierend angesehenen Theorien über Farbenplastik. Er war überzeugt, daß verschieden gefärbte Teile eines Bildes beim Betrachter den Eindruck ganz bestimmter, ungleicher Entfernungen der Bildteile hervorrufen. Er erklärte solch eine vermeintliche «Stereoskopie durch Farbdifferenz» vor allem physiologisch, d.h. aus dem Bau und Sehvorgang der menschlichen Augen. Auf Grund seiner Theorie gelangte er zu einer Höhenfarbenskala, die der Farbfolge eines Teiles der Spektralfarbenreihe annähernd entspricht. Diese Beziehung zu einem physikalischen Phänomen ließ seine Theorie als plausibel erscheinen. Sie ist jedoch nicht haltbar und basiert auf verschiedenen irrigen Annahmen. Gäbe es eine physikalisch oder physiologisch bedingte Stereoskopie durch Farbdifferenz, so würde jede bunte Bildtafel in sinnloser Art räumlich auseinanderfallen. Eine unter gewissen Umständen tatsächlich wahrnehmbare bildliche Farbenplastik ist ausschließlich psychologisch bedingt. Die bildlichen Farbe-Raum-Beziehungen beruhen auf Erfahrung, auf meist unbewußter geistiger Vorstellungsreproduktion. Nur in Verbindung mit irgend welchen figürlichen Gebilden besitzen Farbtöne im Bilde raum- oder distanzgliedernde Wirkung. Gestützt auf eine Reihe von Experimenten hatte ich schon 1925 auf diesen Sachverhalt hingewiesen (Lit. Nr. 1).

Nun besitzen wir in der natürlichen Luftperspektive, wie sie sich zu jeder Zeit in jeder Landschaft zeigt, eine ausgezeichnete Hilfe zur Unterscheidung von nah und fern.

Die atmosphärische Trübung verschleiert weit entfernte Geländeteile, hellt sie auf und läßt sie hellblaugrau erscheinen. Alle, selbst die kräftigsten und buntesten (nicht selbst leuchtenden) Körperoberflächenfarben nähern sich mit wachsender Entfernung diesem Luftschleierton. Kontraste von Hell und Dunkel, von Licht und Schatten und solche von irgendwelchen Farben werden durch die Luftperspektive mit wachsender Distanz mehr und mehr gemildert und die Tonunterschiede schließlich ganz zum Verschwinden gebracht. - Diese alltägliche Erscheinung übertragen wir sinngemäß in die Karte. Bei der Kartenbetrachtung entspricht die Höhe der Nähe und die Tiefe der Ferne. Somit mischen wir unsern Farbtönen nach der Tiefe hin mehr und mehr einen hellen Blau- oder Grauton bei. Vor allem aber steigern wir die Licht- und Schattenkontraste nach der Höhe hin. Dies ist nur möglich, wenn die Grundtöne oder Höhenstufentöne sich nach oben stark aufhellen. Nur auf hellem Grunde sind starke Lichtund Schattenkontraste möglich. Für schattenplastische Reliefkarten eignet sich daher eine Höhenfarbenskala, die stufenweise emporsteigt von mäßig starkem graublauem Grün der Tiefebene bis zu lichtem, ins Weiß übergehendem Gelb der Höhen.

Alle Gebiete über $3000 \mathrm{~m}$, somit alle größeren zusammenhängenden Firn- und Gletschergebiete besitzen einen weißen (farblosen) Grund- oder Höhenton. - 
Auch Peucker beachtete beim Aufbau seiner Höhenfarbenskala luftperspektivische Effekte. Dies und nur dies, nicht aber seine seltsamen physikalischen und physiologischen Theorien, sicherte seiner Farbenskala einen gewissen Erfolg. Er übersah jedoch, daß intensives Rot ein relativ dunkler Farbton ist, der starke Hell-Dunkel-Kontraste, wie sie den Höhen entsprechen, nicht zuläßt.

Wir werden auf unsere Höhenstufenfarben unten näher eintreten. -

\section{3) Abstrakte, mittelbare und konkrete, unmittelbare Abbildung \\ ari}

Eine Gegenüberstellung der früheren und der neuen Alpenländerkarte läßt ihre grundsätzlichen Unterschiede leicht erkennnen.

Die Darstellungselemente der früheren Karte, die Schraffen und die konventionellen Farben, sind ausgesprochen abstrakter oder fiktiver oder symbolhafter Art. Wir können sie auch als mittelbar bezeichnen.

Die Darstellungselemente der neuen Karte sind in höherem Maße unmittelbar bildhaft oder konkret. Sie bringen Unebenheit zu unmittelbarer Anschauung, nicht anders als es im Gemälde des Künstlers der Fall ist. Man zeichnet die Reliefoberfläche (abgesehen von einigen gewollten Verdeutlichungen) so, wie sie beim Anblick eines entsprechend beleuchteten Modelles unmittelbar in Erscheinung tritt.

Der Widerstreit der beiden gegensätzlichen Abbildungsprinzipien besteht - bewußt oder unbewußt - in der Kartographie seit jeher. Am frühesten gelangte eine unmittelbare Darstellung grundrißlicher Geländeformen in Hans KonRad GygER's Zürcher Kantonskarte des Jahres 1667 zur Anwendung. Etwa seit 1880 setzte sie sich in der Schweiz für Karten großer und mittlerer Maßstäbe allgemein durch. Rudolf LEUzinger, Fridolin Becker, Xaver Imfeld und Hermann Kümmerly waren ihre bedeutendsten Bahnbrecher. In neuerer Zeit suchten wir die Reliefkarte zu verbessern und konsequenter zu gestalten. Unter dem Einfluß des photographischen Luftbildes, der erleichterten Modellerstellung und der Modellphotographie erobert sie sich heute rasch auch die Geländekartographie anderer Länder. Ein höheres Maß an Anschaulichkeit, an Bildhaftigkeit, Naturähnlichkeit und wohl auch an Schönheit sind Vorzüge dieser Darstellungsart. In Karten großer und mittlerer Maßstäbe kann jedoch infolge der unabdingbaren geometrischen Ansprüche in der Regel auf eine Verbindung mit dem fiktiven, mittelbaren Element der Höhenkurve nicht verzichtet werden. Nur bei geschickter Kombination mittelbarer und unmittelbarer Bild-Elemente besitzt die Karte die von ihr gewünschte optimale Aussagekraft.

Für Karten kleiner und sehr kleiner Maßstäbe aber galt die schattenplastische, naturähnliche Reliefdarstellung langezeit als undurchführbar oder als ungeeignet. Erst seit einigen Jahren gehen Kartographen verschiedener Länder auch an dieses Problem heran. Die beiliegende Alpenländerkarte $1: 2500000$ ist ein Ergebnis meiner eigenen diesbezüglichen Versuche. -

\section{4) Die Besonderheit des Problems für Karten kleiner Maßstäbe}

Wo liegen die Schwierigkeiten unmittelbarer schattenplastischer und farbig naturähnlicher Reliefdarstellung in Karten kleiner Maßstäbe? Wodurch unterscheidet sich das Problem von demjenigen des Abbildens in großen Maßstäben? Auf diese Fragen sei im Folgenden kurz eingetreten.

Einem Blatt einer Gebirgskarte großen Maßstabes entspricht in der Regel ein topographisches Modell mit relativ großflächigen Formen und beträchtlichen Höhenunterschieden. An einem solchen Modell, wie auch in einer entsprechenden kartenartigen schattenplastischen Modellphotographie sind die markanten Relief-Formen und starken Höhengliederungen leicht wahrnehmbar. Das Motiv oder der Vorwurf ist als Reliefkarte ausgesprochen abbildungsgünstig. 
Nicht so ist es für sehr kleine Maßstäbe. Das einem Kartenblatt entsprechende Landschaftsmodell besitzt stets die Gestalt eines äußerst flachen, äußerst feinknitterigen Fladens. Flache, aber wichtige Geländeschwellen, beträchtliche und bedeutsame Höhenunterschiede weit auseinanderliegender Ebenen sind ohne Meßhilfsmittel selbst am Modell kaum wahrnehmbar. Noch viel weniger ließe die kartenartige Modellphotographie solche Schwellen und Höhenunterschiede erkennen. Die Feinknitterung der Gebirge führt im Abbild leicht zu verwirrender chaotischer Unklarheit und Unübersichtlichkeit. Das Motiv ist als Reliefkarte ausgesprochen abbildungsungünstig.

Um genügende Bildhaftigkeit und Klarheit zu erreichen, sieht sich hier der Kartograph genötigt, die Formen stark zu vereinfachen und zu übertreiben. Ferner muß er die Höhenstufen farbig genügend unterscheiden und somit mehr oder weniger von einer gewissen Naturähnlichkeit abweichen. Jede höhenzonale farbige Zebrastreifung stört aber, wie bereits betont, den schattenplastischen Modelleindruck.

Dazu tritt folgende weitere Erschwerung: In Karten sehr kleiner Maßstäbe und entsprechend großer Gebiete herrschen im allgemeinen ebene Flächen stark vor. Diese aber besitzen bei schräg einfallendem Licht naturgemäß einen leichten Schattenton, einen sogenannten Halbton. Auf diesem verbindenden Halbton der Ebenen basiert für flache Gebiete im Wesentlichen der unmittelbare Reliefeindruck. Dieser graue Halbton aber legt sich auf die Höhenstufenfarbtöne, trübt sie und vermindert ihre Unterscheidbarkeit.

Höhenfarben und Schattentöne sind daher äußerst behutsam zu wählen und sehr sorgfältig aufeinander abzustimmen. Dies gilt nicht nur für die Originalentwürfe, es stellt auch an die Reproduktion hohe Ansprüche.

Solche und andere Dinge seien in den folgenden Abschnitten näher betrachtet.

\section{5) Das schattenplastische Reliefbild}

Das geometrisch geregelte Gefüge der Schraffen zwingt zu starker Vereinfachung der Formen. Dieser Umstand und mehr noch falsche Lehrmeinungen und Vorstellungen führten in den Schraffenkarten kleiner Maßstäbe oft zu naturwidrigen, falschen Terrassen, Wülsten, Rundungen, Kegelgipfeln, raupenähnlichen Hügelformen usw.

Die schattenplastische Reliefzeichnung hingegen ermöglicht eine weitergehende Annäherung an die Naturformen; sie erlaubt eine subtilere Anpassung auch an feingegliederte und an flachwellige, wenig ausgeprägte Formen. Die Gegensätze von Hochgebirge, Mittelgebirge und Hügelland lassen sich besser zum Ausdruck bringen. So erscheinen z. B. die Bruchränder der Oberrheinischen Tiefebene und des Rhone-SaôneGrabens geschlossener und schärfer, als in der Schraffenkarte.

Solche neuen und besseren Möglichkeiten aber verpflichten. Der gestaltende und umformende Kartograph benötigt wesentlich besseres, genaueres topographisches Grundlagematerial, als für die Erstellung von Schraffenkarten entsprechender Maß. stäbe seinerzeit der Fall gewesen war.

Die Anforderungen an das topographische Grundlagematerial hängen ab vom Maßstab der neu zu zeichnenden Karte. Zur Bearbeitung einer Karte 1:2 500000 sollten gute, einheitliche schattenplastisch überarbeitete Höhenkurvenkarten mindestens im Maßstab 1:500000 zur Verfügung stehen. Diese Vorbedingung trifft für den Alpenraum einigermaßen zu, leider aber noch längst nicht für alle Landgebiete der Erde. Wir sind heute noch nicht einmal in der Lage, für alle Gebiete der Erde gute, einheitliche, den Reliefformen wirklich entsprechende schattenplastische Karten im Maßstab 1:4000 $000 \mathrm{zu}$ zeichnen, denn vielenorts fehlen noch auf Jahre und Jahrzehnte hinaus die erforderlichen topographischen und kartographischen Grundlagen. Höhenstufenkarten 1:1000000 von sehr unterschiedlicher Genauigkeit (Internationale Weltkarte) sind heute noch vielenorts die einzigen Quellen. Solche Karten aber, Blätter ohne feinmaschig-aequidistante Höhenkurven und ohne gut und präzis gearbei- 
tetes schattenplastisches Relief, reichen nicht aus. Die Schraffendarstellungen der klassischen Handatlanten des letzten Jahrhunderts aber sind veraltet und zu ungenau. Für einzelne Gebiete liegen neuartige Karten vor, deren Geländedarstellungen durch photographische Aufnahmen von Geländemodellen entstanden sind. Ihre plastischen Effekte erscheinen oft vorzüglich, erweisen sich aber vielenorts als trügerisch, weil die Modelle im Allgemeinen zu ungenau gestaltet sind und weil die Schatteneffekte zu sehr von der Beleuchtungsrichtung abhängen. Es fehlt solchen mechanisch erstellten Schattierungsbildern überdies die so notwendige zusammenfassende Überarbeitung zur Hervorhebung oder Differenzierung der landschaftlichen Großgliederungen.

So kommt es, daß in den meisten großen neuen Handatlanten unserer Zeit das Erdoberflächenrelief nur noch sehr grobmaschig durch farbige Höhenstufen zum Ausdruck gebracht wird. $\mathrm{Zu}$ solchem Verzicht auf subtilere Reliefdarstellungen durch Schraffen oder durch Schattentöne mögen freilich oft auch ökonomische Überlegungen und personelle Schwierigkeiten geführt haben. Die Erstellung guter Reliefschattenbilder für Karten kleiner Maßstäbe (und auch guter Schraffenbilder) ist äußerst zeitraubend und kostspielig. Oft stehen den kartographischen Instituten genügend befähigte Geländezeichner überhaupt nicht zur Verfügung. -

\section{6) Die farbige Höhenabstufung}

Wir haben auf einige Gesichtspunkte der farbigen Höhenabstufung oben bereits hingewiesen. Hier nun sei die in unserer neuen Alpenländerkarte getroffene Lösung näher beschrieben und begründet.

Zunächst sei das bereits Gesagte nochmals zusammengefaßt:

1) Soll die Schattierungsmodulation zur beabsichtigten Wirkung gelangen, so ist die Höhenfarbenskala im Ganzen aus hellen Farben zusammenzufügen.

2) Sollen sich die Schattierungskontraste nach oben luftperspektivisch verstärken, so sind die Höhenfarben insbesondere nach oben stark aufzuhellen. Am hellsten aber sind helles Gelb und Weiß.

3) Die unterste Stufe der farbigen Höhenskala entspricht dem luftperspektivisch verschleierten Tiefland. Die passende Farbe hierfür ist, da es sich um Vegetationsgebiete handelt, offensichtlich ein leichtes grau gedämpftes Blaugrün.

4) Zwischen den beiden Grenzwerten, dem Blaugrün der Tiefe und dem hellen Gelb, resp. Weiß der Höhe, sollen sich die Farbtöne von unten nach oben stetig aufhellen.

Sowohl die oben genannten luftperspektivischen Erscheinungen, wie auch eine gewisse Logik verlangen Beachtung dieser einfachen «je-desto-Beziehung». -

5) Bei Schrägbeleuchtung legt sich ein leichter grauer Halbschatten auf alle Geländeverflachungen und Ebenen. Der entsprechende Schattenton aber trübt die Ḧ̈henfarbtöne und beeinträchtigt ihre Unterscheidbarkeit. Trotzdem wollen wir auf diesen Ebenenton nicht verzichten, denn ohne ihn kann ein unmittelbarer plastischer Reliefeindruck besonders im außeralpinen Gebiet nicht zu Stande kommen. -

6) Die sieben verschiedenen Töne der Höhenfarbskala sollen sich genügend voneinander unterscheiden.

Dies ist in Karten kleiner Maßstäbe notwendiger, als in Gebirgs-Reliefkarten großer Maßstäbe, wo Höhenkurven und kräftige, einfache Schattierungsgliederungen mithelfen, die Reliefgestaltung und damit auch das Hoch und Tief erfassen zu lassen. -

Genügende Unterscheidbarkeit der Höhenfarbtöne ist besonders in den meist flachen Regionen unter $500 \mathrm{~m}$ erwünscht, da hier selbst geringe Höhenunterschiede von großer Bedeutung sind. - 
Diese sechs Postulate lassen sich schwer unter einen Hut bringen, sie stehen sich zum Teil geradezu entgegen. Eine siebenstufige Farbskala, die nur von Blaugrün über Grün zu Gelb und Weiß führt, die überdies im Ganzen stetig und hell ist, und die großenteils durch graue Schattentöne verschleiert wird, läßt kaum eine genügend sichtbare Differenzierung ihrer einzelnen Stufen zu.

Somit, was tun? - Es gibt nur einen einzigen einigermaßen gangbaren Ausweg: Die Skala wird kräftiger differenziert, indem man ihr stellenweise Rot (die dritte der drei Grundfarben) einfügt oder beimischt.

Dies aber kann auf drei Arten geschehen:

Erste Art: Man läßt das Gelb nach oben durch Beimischung von Rot in leichte rötliche Töne übergehen. Dies war der bisher meist eingeschlagene Weg. Die Höhenfarben vieler Gebirgs-Reliefkarten großer Maßstäbe sind so abgestuft. Hierbei ist das beigemischte Höhenrot (oder Höhenrosa) äußerst leicht (hell) zu wählen. Rot ist eine dunklere Farbe als Gelb. Starkes Rot verdunkelt in einer schattenplastischen Karte vor allem die Lichthänge. Ist die Rotbeimischung äußerst leicht, so macht sich solche Verdunkelung kaum störend bemerkbar. Das Rosa oder Orange in ein Gitter brauner Höhenkurven oder brauner Felsgerippelinien eingebettet, ergibt einen leicht bräunlich erscheinenden, felsfarbenen Bodenton. Dies ist für Gebirgs-Reliefkarten großer Maßstäbe ein offenbarer Vorzug, denn ausgedehnte reine Gelbflächen wären weder sehr naturähnlich, noch ästhetisch befriedigend.

Diese soeben geschilderte Höhenfarbenabstufung, wie sie sich in alpinen, schattenplastischer Reliefkarten großer Maßstäbe oft findet ${ }^{1}$, stand auch für unsere neue Alpenländerkarte 1:2500000 zur Diskussion. Doch erschien sie mir nicht als die beste Lösung. Versuche zeigten, daß man in Anbetracht der Kleinheit und Isoliertheit der hochgelegenen Flächen und beim Fehlen der farbunterstützenden Höhenkurven eine genügende Unterscheidbarkeit der Farben nur erreicht hätte durch wesentlich kräftigere Rotbeimischung. Damit aber hätten wir uns auf einem Circulus vitiosus herumbewegt. Entgegen den oben dargelegten Prinzipien wären die höchsten Gebiete durch das Rot verdunkelt und in ihren Reliefwirkungen geschwächt worden, das helle Gelb wäre wieder in die Mittellage der Höhenfarbskala gerückt. Wir wären bei einer Skala angelangt, die sich der traditionellen, für unsern $Z_{\text {weck }}$ ungeeigneten Farbreihe der bisherigen Alpenländerkarte angenähert hätte. -

Zweite Art: Man hätte eine deutlichere Farbdifferenzierung nach unten hin prüfen können, so etwa durch Weglassen der Gelbbeimischung im Tieflande? Der hierbei verbleibende helle Blauton aber wäre als Tieflandton völlig ungeeignet, er bleibt in der Karte den Wasserflächen vorbehalten. -

Oder indem man das Tieflandgrün durch Beimischen von Rot weiter variiert hätte? Auch dies geht nicht. Grün und Rot, resp. Blau und Gelb und Rot ergeben je nach ihren Mischungsverhältnissen bräunliche oder graue Töne. Bräunliche Töne in der Tiefe, resp. Ferne widersprechen den natürlichen luftperspektivischen Erscheinungen. Grau als Tieflandton, als Basiston gleichsam, kann bei geschickter Farbwahl ausgezeichnet wirken. Schon Rudolf LEUZINGER verwendete manchmal Grau für seine untersten Stufen, ebenso später KarL PEUcker. Wir prüften diesen Weg ebenfalls, doch kamen wir wieder davon ab, weil hypsometrische (höhenzonale) Grautöne und die ebenfalls grauen Schattentöne sich zu ähnlich sind. Durch ihre Kombination entsteht Unklarheit, Unschärfe. Reliefformen und Höhenlagen werden verwischt, statt differenziert und geklärt. Überdies widerstrebt es uns, die grünen fruchtbaren Tiefebenen des Po, der Rhone und des Rheines durch steriles totes Grau darzustellen. -

1 Beispiel: Karte Jungfraugruppe und Aletschgletscher 1: 100000 im Schweizerischen Mittelschulatlas, Ausgaben 1932 bis 1958 (ff). 
Dritte Art: Eine reichere Differenzierung unserer Höhenfarbenskala kann auch erreicht werden durch leichte Rotbeimischung in den Mittellagen. Diese Lösung findet sich in unserer neuen Alpenländerkarte. Je nach den Mischungverhältnissen resultieren aus Blau, Gelb und Rot olivgrüne oder bräunliche Töne. Diese werden in unsere Farbskala eingefügt. Wir erhalten damit folgende sieben Abstufungen:

$$
\begin{aligned}
0-100 \mathrm{~m} & \text { Blaugrün } \\
100-200 \mathrm{~m} & \text { Grünoliv, leicht } \\
200-500 \mathrm{~m} & \text { Braunoliv, leicht } \\
500-1000 \mathrm{~m} & \text { Braun, sehr leicht } \\
1000-2000 \mathrm{~m} & \text { Orange, leicht } \\
2000-3000 \mathrm{~m} & \text { Gelb } \\
\text { über } 3000 \mathrm{~m} & \text { Weiß }
\end{aligned}
$$

Um eine Störung der Schattierungsmodulation zu vermeiden, sind alle diese Höhenstufentöne leicht gehalten, wobei sie sich von der Tiefe zur Höhe aufhellen. Ein solches Aufhellen ist nur möglich, wenn die unterste Stufe, das Blaugrün unter $100 \mathrm{~m}$, etwas kräftiger (dunkler) gewählt wird, als sich aus landschaftlich-luftperspektivischer Schau ergäbe. Die weiße Stufe, diejenige über $3000 \mathrm{~m}$, liegt in der Region ewigen Schnees. Der weiße Ton zeigt somit hier zugleich Höhenlage und Schneebedeckung. -

\section{7) Das Zusammenspiel von Höhenfarbtönen und Schattentönen}

Soll das Gesamtbild nicht zerfallen, so müssen die Höhenstufenfarben und die Schattentöne sowohl in den Farbwerten, wie in den Tonstärken harmonisch zusammenspielen. Höhentöne und Schattentöne sind zwei grundsätzlich ganz verschiedene Dinge. Die Höhen-Farbtöne sollen möglichst rein sein (keine Grau-Anteile enthalten), da sie ohnehin durch die aufgelagerten grauen Schattentöne gedämpft werden. Die Schattentöne aber sollen weder manierhaft bunt, noch stumpf aschengrau sein, sondern natürlichen blaugrauen Schattentönen entsprechen. In der von Ferne gesehenen sonnigen Landschaft tritt der Wechsel von Licht und Schatten viel mehr in Erscheinung, als der Oberflächen-Farbton. So soll es auch in der naturähnlichen Reliefkarte sein. Die Modulation durch Licht und Schatten ist für das Zustandekommen eines Formeindruckes viel entscheidender, als die Oberflächenfarben. Die leichteren Schatten des Tieflandes und der Hügelregion sollen, den luftperspektivischen Effekten entsprechend, einen ausgesprochenen Einschlag von Blau besitzen. Ein leichtes blaues Grau fügt sich mit dem Höhengrün der Tiefebenen zu einem leicht gedämpften schönen, genügend bunten Ton. Rötliche oder aber unbunte aschgraue Schattentöne, etwa in der Art von Bleistifttönen, würden die Höhenfarbtöne zu sehr verschmutzen. Anders verhält es sich mit den starken Schatten der höheren alpinen Bergkämme. Diese treten nur in sehr kleinen Flächen auf und sie decken ihren Untergrund ohnehin völlig. Solch starke Schatten sollen stärkeren Rotgehalt aufweisen, ihr Ton soll ein frisches grau-gedämpftes Violett sein. Solche Nüancierungen der Schattentonfarben von der Tiefe (Ferne) zur Höhe (Nähe) entprechen ebenfalls den luftperspektivischen Erscheinungen in der Landschaft.

\section{8) Zeichnungs- und reproduktionstechnische Vorgänge und Probleme}

Jede neue Kartenform birgt in sich neue Probleme der Zeichnungs- und Reproduktionstechnik. So verhält es sich auch im vorliegenden Falle der neuen Alpenländerkarte. Einige solche Fragen, soweit sie den geographischen Leser interessieren mögen, seien im Folgenden kurz angedeutet.

Die Schraffendarstellung, ursprünglich ein Kind des Kupferstiches, gilt heute als überlebt. Sie wurde verdrängt durch das rationellere, raschere, leichtere Schummern und Schattieren. Sie gilt so sehr als überlebt, daß jüngere Kartographen ihre zeichne- 
rische oder gravierende Erstellung gar nicht mehr lernen. Die Schraffen-Kartographen sind am Aussterben. Dies ist bedauerlich, denn auch heute noch wäre in manchen Fällen eine gut gezeichnete Geländeschraffe das geeignete Darstellungselement. Die allzu frühe Verdammung hat die Schraffe wohl weniger ihrer mangelnden Ausdruckskraft, als vielmehr der Mühseligkeit und Schwierigkeit ihrer Erstellung zuzuschreiben. Für uns aber ist das Ausscheiden der Schraffentechnik mit ein Grund, die sie ersetzenden Schummer- und Schattentechniken möglichst zu vervollkommnen. Die Entwicklung ist hierin noch nicht abgeschlossen.

Noch vor wenigen Jahrzehnten malte man Reliefkartenoriginale in den endgültig gewünschten Gliederungen und Farben. Die einzelnen Farbelemente wurden hierauf manuell durch den Chromolithographen auf die verschiedenen Druckplatten übertragen.

In neuerer Zeit trennt man oft schon bei der zeichnerischen Erstellung die verschiedenfarbigen Inhaltselemente. Man erstellt mehrere, den verschiedenen Druckfarben entsprechende Einzeloriginale. Die Licht- und Schattenmodulation entsteht hierbei als Weiß-Schwarz-, resp. Grau-Original, das dann photographisch unter Verwendung von Rastern auf die Druckplatten gebracht werden kann. Für die Höhenstufenfarben werden gesonderte Vorlagen erstellt. Der Kartenbearbeiter sieht hierbei die farbig kombinierte Karte erstmals erst in der Form von Druckproben. So entstand u.a. die vorliegende Alpenländerkarte.

Dieser Weg ist rationeller und führt oft zu formtreuerer Reproduktion, weil Auge und Hand des nachahmenden Chromolithographen ausgeschaltet sind. Auch bei diesem Verfahren zeigen sich indessen Schwächen oder Schwierigkeiten, die heute noch nicht behoben sind. Auf einige derselben sei im Folgenden hingewiesen:

1) Das schattenplastische Reliefbild entsteht als Grau-Original, das im Interesse leichterer Reproduzierbarkeit möglichst kontrastreich gestaltet wird, d.h. alle Abstufungen von Weiß bis zu Schwarz erhält.

Drucktechnisch aber lassen sich die verschiedenen Schattierungsabstufungen nicht mittels einer einzigen Druckplatte, resp. durch einen einzigen Druckvorgang wiedergeben. Es sind zwei (in manchen Karten drei) verschiedene Schattenplatten, resp. Druckgänge erforderlich. Ein «erster Schummer» wird heller und blauer, ein zweiter verstärkender Schummer meist etwas kräftiger und violetter gedruckt. Die Grau-Abstufungen des Originals müssen somit durch besondere photographische Manipulationen auf zwei oder drei Bilder, resp. Druckplatten zerlegt werden. Durch die zwei oder drei Druckgänge werden dann diese zwei oder drei Bilder wieder zu einem einzigen addiert. Es gibt heute noch keinen genügend einfachen und genügend sicheren Weg, solche Grauton-Separation und Wieder-Addition völlig konform durchzuführen. Das gedruckte Schattierungsbild weicht daher immer noch etwas ab von den Modulationseffekten des Originals.

Mit der genannten Separation und Wieder-Addition ist die folgende weitere Schwierigkeit verknüpft: die verschiedenen Drucke müssen auch lagemäßig aufs Allerschärfste zusammenpassen. Bei der Feinzerknitterung der Reliefformen und der entsprechenden Kleingliedrigkeit der Schattenflecken, wie sie die kleinen Maßstäbe mit sich bringen, würde sich ungenaues Passen viel verheerender auswirken, als in Reliefkarten großer Maßstäbe. -

2) Dem Kartenersteller stehen aus Gründen möglichster Kostensenkung nicht beliebig viele Druckfarben zur Verfügung. Insbesondere ist für die Karten eines Atlas eine bestimmte, möglichst kleine Anzahl gut gewählter Druckfarben im vornherein festgelegt. Reliefkarten, geologische Karten, Niederschlagskarten, Volksdichtekarten, Produktekarten usw. müssen mit denselben Farben gedruckt werden. Beispielsweise standen für die Alpenländerkarte in der Form, wie sie diesem Aufsatze beigegeben ist, nur die folgenden sechs Druckfarben zur Verfügung: 
1) Kräftiges Blau für die Gewässerlinien

2) Helles Blau für Wasserflächen und Höhenstufentöne

3) Gelb für Höhenstufentöne

4) Rosa (helles Rot) ebenfalls für Höhenstufentöne

5) Blaues Grau als ersten Schattenton

6) Grauviolett als zweiten Schattenton

Mit diesen Druckfarben war das vorliegende farbige Bild aufzubauen.

In der fertig gedruckten Karte, wie sie im Schweizerischen Sekundarschulatlas (Ausgabe Frühjahr 1959) enthalten ist, treten folgende weitere Druckfarben hinzu:

7) Dunkelbraun für Signaturen, wie z. B. Ortszeichen und Autobahnen

8) Kräftiges Rot für die Eisenbahnlinien

9) Kräftiges Grün für Landesgrenzen-Bänder

10) Schwarz für die Kartenbeschriftung

Schönheit und Klarheit des Reliefbildes werden durch die Eintragung der Signaturen, Grenzbänder, Namen, Höhenkoten usw. empfindlich gestört. Dies läßt sich nicht völlig vermeiden und gilt für jede Karte. Andererseits aber werden gerade im vorliegenden Falle die in der Karte vorherrschenden milden Blau-Grün-Gelb-Flächentöne durch das mit ihnen kontrastierende Rot der Bahnlinien angenehm belebt. Jeder Kartenersteller hat solchen Fragen des Zusammenspiels seine ganz besondere Aufmerksamkeit zu schenken. -

3) Es gelang erst nach längerem Bemühen mit den oben genannten Druckfarben die erforderlichen äußerst subtilen Abstufungen oder Nüancierungen der Töne unserer Karte zu drucken. Die mittleren Stufen unserer Farbskala enthalten - in ungleicher Dosierung und somit in verschiedenen Rasterstufen - Blau, Gelb und Rosa. In allen Höhenstufen treten überdies an Schattenhängen und auf Verebnungen Grautöne hinzu, deren Dosierungen, resp. Rasterungen ebenfalls aufs Feinste abzuwägen waren. (Die Tiefebenen z. B. dürfen keine Spur des zweiten Schummers und auch den ersten Schummer nur als leichten Halbton enthalten, so daß durch dessen Addition mit dem Tieflandgrün ein Ton resultiert, der immer noch leichter erscheint, als derjenige der aus den Ebenen aufsteigenden Schattenhänge). - Die erhofften Ergebnisse konnten nur erreicht werden durch Verwendung sehr feiner und gut abgestufter Raster und durch manuelle Retouchen der Rasterbilder in den Filmpositiven (Entfernen der Punkte in den hellsten Lichtern und Decken der tiefsten Schattenpartien). -

Weitere Einschränkungen der Druckfarbenzahl ohne «Gesichtsverlust» der Karte wären theoretisch durchaus denkbar, da sich bekanntlich durch drucktechnische Addition bestimmter Mengen der drei Grundfarben Blau, Gelb und Rot alle beliebigen Farbtöne drucken lassen. Es wären hierzu äußerst reine, spektrale Druckfarben, äußerst feine und gut abgestufte Raster, äußerst exaktes Passen, bestes Druckplattenmaterial und besondere Qualitätspapiere erforderlich. Versuche solcher Art führten in der Kartographie bisher noch nicht zu den gewünschten Ergebnissen. Sowohl die besondere graphische Struktur der Karte (die nur ihr anhaftende Verbindung von Farbflächen mit feinsten verschiedenfarbigen Linien und Signaturen), wie auch die Anforderung ihrer immer wiederkehrenden leichten Nachführung, erschweren das Problem.

\section{9) Einige abschließende Betrachtungen}

In der neuen Alpenländerkarte sind wohl erstmals in kleinem Maßstab konsequent durchgeführte Schräglicht-Schattierungen und luftperspektivische Höhenfarben- und Kontrastabstufungen miteinander vereinigt, und zwar so, daß sich die verschiedenen Elemente gegenseitig möglichst wenig stören. 
Eine solche Synthese von Reliefformenbild und naturähnlicher Höhenfarbengliederung (soweit man in einem 2500000 mal verkleinerten Landschaftsbilde überhaupt von Naturähnlichkeit reden kann) führt zu optimal möglicher Anschaulichkeit, zu optimal möglicher visueller Vermittlung landschaftlicher Vorstellungen. Darin liegt der wesentlichste, aber nicht der einzige Gewinn der neuen Darstellungsart. Einige weitere Vorzüge sind:

1) Die Reliefwiedergabe ist nicht nur anschaulicher, sondern auch genauer und charakteristischer, dennoch aber ruhiger, als bei den traditionellen Schraffen- und Schummerdarstellungen.

2) Der farbige Aufbau der Höhenstufen ist stetiger, als bei den traditionellen Farben. Der irrige Eindruck eines treppenförmigen landschaftlichen Aufbaues, wie er sich aus bisherigen Karten oft ergibt, wird damit vermieden.

3) Die unlogische und visuell unrichtige optimale Aufhellung der Höhenfarben in der Skalenmitte ist vermieden, da die helle Gelbstufe an das obere Skalenende gesetzt ist.

4) Die deutlichsten Farbdifferenzierungen erfolgen bei den unteren und mittleren Stufen, denjenigen unter $1000 \mathrm{~m}$. Dies erscheint sinnvoll und zweckmäßig, da in Höhen über $1000 \mathrm{~m}$ jegliche, auch noch so kräftige Höhenfarbendifferenzierung infolge der Gebirgszeichnung ohnehin nicht zur Geltung käme. Die Höhenstufenabgrenzungen sind selbst bei der abstrakten kräftigen Farbdifferenzierung bisheriger Karten in $\mathrm{Hö-}$ hen über $1000 \mathrm{~m}$ kaum zu erkennen, da sie hier durch das dichte Gestrüpp des Schraffen- oder Schummerbildes verschleiert werden.

5) Punktartige und lineare Kartenelemente, wie Gewässerlinien, Ortssignaturen, Grenzlinien usw., und auch Namen und Höhenkoten heben sich von flächentonigem Grund besser $a b$, als vom Strichgefüge der Schraffen, sie sind daher in der neuen Karte leichter lesbar. In Zukunft können sie, der feineren Modellierung des Reliefs entsprechend, ebenfalls etwas feiner gezeichnet werden.

Solchen Vorzügen stehen folgende Nachteile oder Bedenken gegenüber:

Die farbige Differenzierung der Höhenstufen ist weniger ausgeprägt, als bei den abstrakten Höhenfarbenskalen. Der Kartenleser hätte gern beides, sowohl ein schönes, plastisches Reliefbild, wie auch deutlich unterscheidbare Höhenstufenfarben. Die Karte aber kann stets nur die eine oder andere Erscheinung mit voller Deutlichkeit zeigen.

Wir glauben, daß für eine Alpenländerkarte, selbst wenn ihr Maßstab sehr klein ist, das Reliefbild von primärer Bedeutung ist. -

Die meisten Urteile über graphisshe Dinge, auch über geo-graphische und kartographische Dinge, sind durch Angewöhnung beeinflußt. Man soll aber Karten nicht nur mit Karten vergleichen, sondern vor allem mit der Natur. Den Schweizern ist die mehr oder weniger naturähnliche farben- und schattenplastische Reliefkarte längst vertraut. Ein erfahrener deutscher Lehrmittelverleger aber gestand mir einst, er würde es nicht wagen, den Schulen in Deutschland solche Karten anzubieten, da man dort allgemein an die traditionellen, abstrakten Grün-Gelb-Braun-Rot-Höhenstufen gewöhnt sei. Mir scheint, daß der brave Mann das Opfer seiner eigenen, persönlichen Angewöhnung war. In der Schweiz fand ich bisher nur einen einzigen Menschen, der für die traditionelle Form eintrat. Das war auch ein Berufsdeformierter, nämlich ein Geograph!

\section{BENÜTZTE ODER ZITIERTE LITERATUR}

1. ED. Імноғ: Die Reliefkarte. Beiträge zur kartographischen Geländedarstellung. In: Jahrheft 1924 der Ostschweiz. Geogr.-Commerz. Gesellschaft St. Gallen. St. Gallen 1925. 92 S., 7 Taf. m. 25 Abb., Schrift. - 2. ED. ImноF (Zeichnung u. Bearbeitung): Schweizerischer Mittelschulatlas. 
Hrsg. v. d. Konferenz der Kantonalen Erziehungsdirektoren. - Zürich: Kantonaler Lehrmittelverlag 1932 (ff.) 12. Auflage 1958. Druck: Art. Institut Orell Füssli, Zürich. (auch franz. und ital.). 3. ED. ImHoF (Bearbeitung und Zeichnung): Schweizerischer Sekundarschulatlas. Hrsg. von der Erziehungsdirektion des Kantons Zürich. - Zürich: Kantonaler Lehrmittelvertrag 1934 (ff.) 8. Auflage 1959. Druck: Art. Institut Orell Füssli, Zürich. - 4. ED. ImHof: Der Schweizerische Mittelschulatlas. In: Geographica Helvetica. Bern. Jg. 3, 1948. S. 293-375, 16 A hb., Schriftt. - 5. Ed. IMHof: Der Schweizerische Mittelschulatlas und die an unseren Schulen verwendeten Unterstufenatlanten. In : Archiv für das Schweizerische Unterrichtswesen. Frauenfeld. Jg. 43, 1957. S. 78-104, Schrift. 6. O. Peschel: Die Erdkunde als Unterrichtsgegenstand. In: Deutsche Vierteljahrsschrift. Jg. 1868. S. 436. zitiert auch in: - 7. K. C. Rothe und E. Weydrich: Der moderne Erdkunde-Unterricht. Wien und Leipzig: Deuticke 1912. - 8. K. PeuCKer: Schattenplastik und Farbenplastik. - Beiträge zur Geschichte und Theorie der Geländedarstellung. - Wien: Artaria 1898. 129 S., 7 Abb., Schrift. 9. K. Peucker: Höhenschichtenkarten, Studien und Kritiken zur Lösung des Flugkartenproblems. In: Zeitschrift für Vermessungswesen. Stuttgart. Bd. 39, 1910.

\title{
UNE NOUVELLE CARTE DES ALPES
}

La nouvelle carte des pays alpestres, à l'échelle de 1:2500000, qui a été dressée en collaboration par l'auteur et l'institut artistique Orell Füssli montre, pour la première fois sur des cartes à petite échelle, une nouvelle manière de représenter le terrain. Cette méthode est prévue pour les cartes de pays et de continents des futures éditions de l'Atlas scolaire suisse. Le dessin en hâchure qui avait été utilisé jusqu'ici pour les cartes est remplacé par une représentation plastique en ombres plus intelligible et plus naturelle. Au lieu des habituelles couleurs abstraites des différents niveaux d'altitude, il apparaît des tons plus clairs et plus semblables aux paysages. On évite ainsi la forte déformation de l'image du relief que provoquent les couleurs des anciennes cartes.

Notre article traite des difficultés et problèmes particuliers à l'application de cette solution aux cartes à petite et très petite échelle. Les questions de l'illustration abstraite et concrète, de la plastique de couleurs, du jeu des divers éléments sont successivement examinés, de même que celles des processus techniques de dessin et de reproduction. Enfin, les avantages et désavantages des deux méthodes, l'ancienne et la nouvelle, sont confrontés.

\section{DER SPÄTGLAZIALE VORSTOSS DES GLÄRNISCHGLETSCHERS}

\author{
RenÉ HantKe
}

Seit 1947 führten C. Schindi.ER und der Verfasser im weitern Glärnischgebiet geologische Kartierungen durch. Diejenigen des ersteren bestätigten (1959) weitgehend die vom Verfasser neulich dargelegten Ausführungen über den spätglazialen Vorstoß des Glärnischgletschers (1958). Immerhin erlauben die Neuaufnahmen C. ScHINDI.ER's diesen Vorstoß noch etwas zu präzisieren.

C. Schindler konnte feststellen, daß die Moränen am Ausgang des Klöntales, im Gebiet Hinter Sackberg-Hochwald, $3 \mathrm{~km}$ WSW von Glarus, weit ausgedehnter sind, als sich dies J. Oberholzer (1900, 1933) vorstellte. J. Oberholzer (1900: 58) glaubte, daß die Moränen von Chälen (Hinter Sackberg-Hochwald) einem Gletscherstand zuzuordnen sind, als «der Seitengletscher des Klönthals gegenüber dem StammLinthgletscher bei Glarus siegreich das Feld behauptete». ED. BRücKNER (1909: 634) zieht die Möglichkeit in Betracht, daß die Chälenmoräne versackt sein könnte und daher heute auf sekundärer Lagerstätte liegen würde. Später weist J. Ober holzer (1933: 484) die Moränen von Chälen dem Bühlstadium (Rapperswil-Hurden) zu. Diese Zuordnung kann jedoch kaum zutreffen, da das Eis zur Zeit des Bühlstadiums (heute Ammersee-Stadiums) im Klöntal viel höher stand, reichte doch damals der Linthgletscher im Raum von Glarus noch bis gegen $1000 \mathrm{~m}$ hinauf. Überdies konnte schon J. Oberholzer (1900: 61) in Glarus Moränen feststellen, deren Material aus 
